\title{
A Mode-locked Fiber Laser Generating High Purity Cylindrical Vector Beams Based on A Mode Selective Coupler in A Figure-8 Cavity
}

\author{
Jie Wang ${ }^{1}$, Hongdan Wan ${ }^{1, *}$, Yu Cai ${ }^{1}$, Zuxing Zhang ${ }^{1,2}$, Bing Sun ${ }^{1}$, Lin Zhang ${ }^{1,2}$ \\ 1 Advanced Photonic Technology Lab, Nanjing University of Posts and Telecommunications, \\ Nanjing 210023, China \\ 2 Aston Institute of Photonic Technologies, Aston University, \\ Birmingham B4 7ET, UK \\ hdwan@njupt.edu.cn
}

\begin{abstract}
We propose and demonstrate an all-fiber passively mode-locked laser with pulsed cylindrical vector beam output based on a mode selective coupler (MSC) in a figure-8 cavity, The MSC made of a two mode fiber and a standard single mode fiber is used as both the intracavity transverse mode converter and mode splitter. Through adjusting the polarization state in the laser cavity, both radially and azimuthally polarized beams have been obtained with high mode purity which are measured to be $>94 \%$. The mode-locked laser pulses have duration of $17 \mathrm{~ns}$ and a repetition rate of $0.66 \mathrm{MHz}$, operating near a wavelength of $1556.3 \mathrm{~nm}$ with a spectral bandwidth of $3.2 \mathrm{~nm}$.
\end{abstract}

Keywords: Lasers, fiber; Lasers, pulsed; Mode-locked lasers

\section{INTRODUCTION}

Cylindrical vector beam (CVB) lasers are receiving increasing attention from various applications, such as surface plasmon excitation [1], optical tweezers [2], and material processing [3], etc, due to their symmetrical polarization and amplitude. CVBs are classified as azimuthally polarized, radially polarized, and hybridly polarized beams according to the spatial distribution of the polarization, especially, tighter focal spots can be obtained by using radial polarization owing to the existence of a strong and localized longitudinal field component [4].

Various kinds of techniques are reported for CVBs generation, such as axial birefringent components, spatial light modulators and interferometric methods. As compared to the solid-state lasers, the all-fiberized structure design eliminates the need for complicated alignment of free-space optical components and thus simplifies the laser architecture, contributing to the compactness and ruggedness of the fiber laser [5, 6]. In Reference [5] we demonstrated a single-longitudinal-mode fiber ring laser with continuous wave (CW) CVB emission using a two-mode fiber Bragg grating as a transverse mode selector. Recently, pulsed CVB fiber lasers have been reported, using mode-locking or Q switching method [7, 8]. Most of these research use offset splicing (OSS) method to generate high-order mode and few-mode fiber Bragg grating (FM-FBG) as the transverse-mode selector. The FM-FBG is necessarily used to separate the fundamental mode and the higher order modes since they transmit in the same fiber. However, high order modes are excited by lateral misalignment between single-mode fiber (SMF) and few-mode fiber (FMF) which introduces insertion loss into fiber cavity and deteriorates slope efficiency of the CVB lasers. Efforts are still needed to improve the efficiency of fiber lasers with CVB emission.

In this paper, we report a high efficiency, passively mode-locked CVB fiber laser based on an all-fiber mode selective coupler (MSC) with higher mode conversion efficiency. To the best of our knowledge, this is the first report on using MSC as the transverse mode converter and splitter in a figure- 8 fiber laser cavity for generation of mode-locked CVB pulses with high mode purity.

\section{THEORETICAL ANALYSIS AND EXPERIMENTAL SETUP}

Figure 1 shows the schematic of the MSC, composed of a two mode fiber (TMF, core/cladding diameter $=19.7 / 125 \mu \mathrm{m}, \mathrm{NA}=0.12)$ and a SMF (SMF-28). The principle of this coupler is to phase match a high order mode in the TMF with the fundamental mode in the SMF, and then to achieve mode conversion [9].

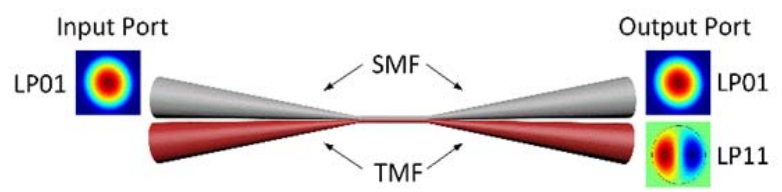

Fig. 1. Schematic of the MSC. The LP01 mode is launched into the SMF input port, the LP11 mode is expected to be excited at the TMF output port, while the uncoupled LP01 mode will propagate along the SMF.

According to the coupling mode equation: 


$$
\begin{aligned}
& \frac{d A_{1}(z)}{d z}=i\left(\beta_{1}+C_{11}\right) A_{1}+i C_{12} A_{2} \\
& \frac{d A_{2}(z)}{d z}=i\left(\beta_{2}+C_{22}\right) A_{2}+i C_{21} A_{1}
\end{aligned}
$$

Where $\mathrm{z}$ is the length of the coupling region. $\mathrm{A}_{1}$ and $\mathrm{A}_{2}$ are the modal field amplitudes of the fundamental mode (LP01) in the SMF and a certain high-order mode in the FMF, respectively. $\beta_{1}$ and $\beta_{2}$ are the propagation constants of the fundamental mode in the SMF and a certain high order mode in the FMF, respectively. $\mathrm{C}_{11}$ and $\mathrm{C}_{22}$ are self-coupling coefficients, $\mathrm{C}_{12}$ and $\mathrm{C}_{21}$ are mutual-coupling coefficients. The self-coupling coefficient is negligible with respect to the mutual-coupling coefficient and approximately $\mathrm{C}_{12}=$ $\mathrm{C}_{21}=\mathrm{C}$. The optical power of the two output ports of the MSC are calculated as:

$$
\begin{aligned}
& P_{1}(z)=\left|A_{1}(z)\right|^{2}=1-F^{2} \sin ^{2}\left(\frac{C}{F} z\right) \\
& P_{2}(z)=F^{2} \sin ^{2}\left(\frac{C}{F} z\right)
\end{aligned}
$$

Suppose $\beta 2$ is the propagation constant of the LP11 mode in the TMF, only if $\Delta \beta=\beta_{1}-\beta_{2}$ is zero, the LP01 mode in the SMF and the LP11 mode in the TMF meet the phase match condition. Then, Eqs. (3) and (4) are: $\mathrm{P}_{1}=\cos ^{2}(\mathrm{Cz})$ and $\mathrm{P}_{2}=\sin ^{2}(\mathrm{Cz})$ indicating a complete periodic power exchange between the two modes in the lossless case. So, the MSC functions both as a mode converter (from the LP01 mode in the SMF to the LP11 mode in the TMF) and a mode splitter by outputting different modes at different fiber output ports.

The propagation constant $\beta 2$ can be calculated as: $\mathrm{n}_{\mathrm{eff}} \mathrm{k}_{0}$, where $\mathrm{k} 0$ is the propagation constant in vacuum, $\mathrm{n}_{\text {eff }}$ is the mode effective index, which varies with the diameter of the fiber. As shown in Fig. 2, we calculated the mode effective index at different fiber diameters for step index profiles by finite element method (FEM). For phase match condition, the best diameter ratio of the SMF as compared to the TMF is about 0.63 , so the diameter of the SMF should be pre-tapered to $79 \mu \mathrm{m}$.

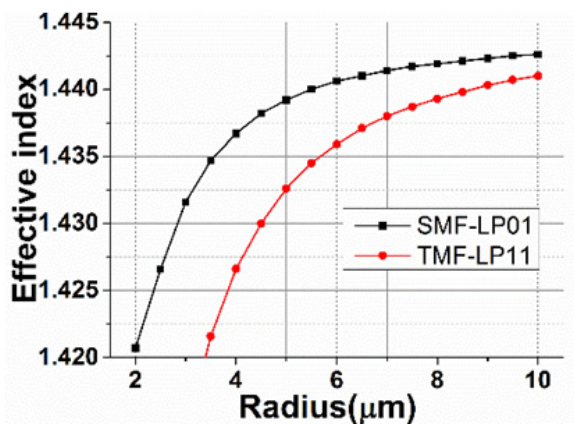

Fig. 2. The mode effective index of the LP01 mode (in the SMF) and the LP11 mode (in the TMF) versus different fiber radius at the wavelength of $1550 \mathrm{~nm}$.

Then, we use beam propagation method (BPM) to calculate and confirm the high efficiency modal power exchange under the phase match condition. As shown in Fig. 3, when the diameters of SMF and TMF optical fibers cores are $5.1 \mu \mathrm{m}$ and $8 \mu \mathrm{m}$, respectively (the diameter ratio is about 0.63 ), the phase match condition is satisfied and only the LP11 mode is excited in the TMF with high mode conversion efficiency.
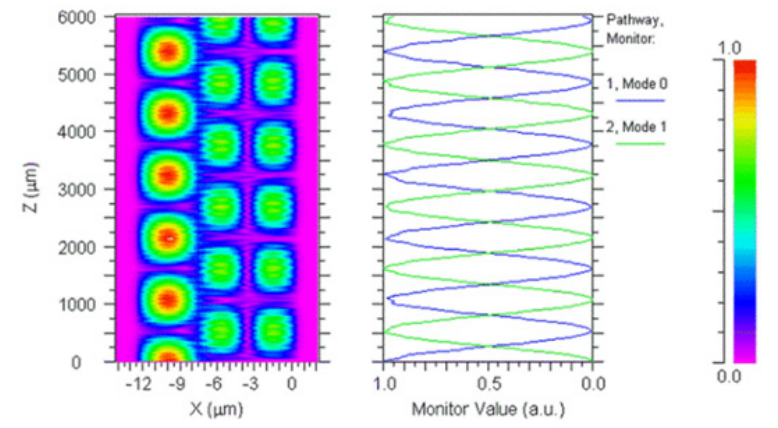

Fig. 3. Simulation of (Left) mode intensity distribution in the fiber; (Right) The power exchange in the coupling region. The LP01 mode in the SMF is converted to LP11 mode in the TMF at the wavelength of $1550 \mathrm{~nm}$.

According to our simulation results, the SMF is pre-tapered to about $79 \mu \mathrm{m}$, then carefully aligned with the TMF and fused them together using the modified flame brushing technique. A laser source of $1550 \mathrm{~nm}$ is launched into the SMF input, the output power of SMF and TMF are detected simultaneously by power meter. As shown in Fig. 4, the mode field distributions at the TMF output port at different wavelengths are detected by a CCD (CinCam IR). The purity of the LP11 mode is estimated to be about $97 \%$ near wavelength of 1550 $\mathrm{nm}$. The LP01 mode can be efficiently converted to the LP11 mode with a low insertion loss of about $0.65 \mathrm{~dB}$.

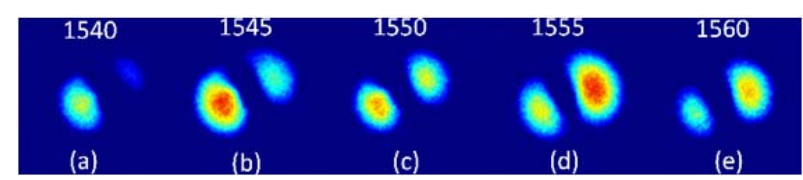

Fig. 4. CCD images of the LP11 mode excited in the TMF at different launching wavelengths.

\section{EXPERIMENTAL RESULTS AND DISCUSSIONS}

The experimental setup of the proposed all-fiber mode-locked CVB laser is shown as in Fig. 5. The MSC is inserted into the figure- 8 cavity (right section) between a 50:50 fiber coupler and a 90:10 fiber coupler. The figure- 8 cavity consists of a $980 \mathrm{~nm}$ laser diode, a 980/1550 nm wavelength division multiplexer (WDM), a section of $\mathrm{Er}^{3+}$-doped fiber (EDF), three polarization controllers (PCs), a long section fiber (550m, SMF28e+) and an isolator (ISO). The mode-locking mechanism Amplifying Loop Mirror (NALM), through adjusting the PC1 in the cavity.

The proposed fiber laser uses MSC instead of FM-FBG as the transverse mode converter and eliminates extra cavity loss. The insertion loss of the MSC is $0.65 \mathrm{~dB}$ with an intra-cavity coupling ratio of $90 \%$. The spectral and temporal properties of the laser are measured at output1, by an optical spectrum analyzer (OSA, YOKOGAWA, AQ6370C) and an oscilloscope (OSC, SDA 6000A). The output beam profiles are captured by a CCD camera placed at output2 through a fiber collimator. 


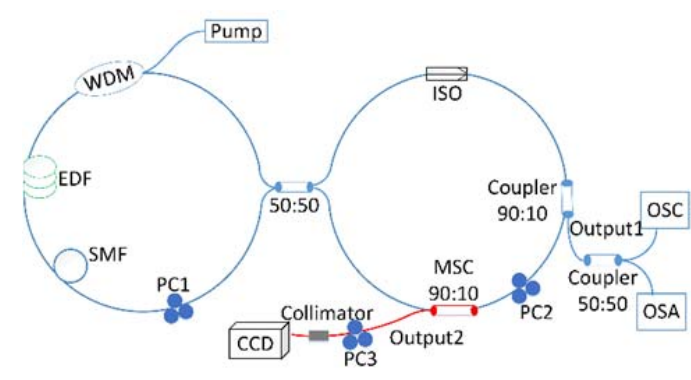

Fig. 5. Experimental setup of the all fiber passively mode-locked CVB laser and monitoring system

Stable pulse trains can be observed by adjusting the PC1 in the cavity. For a pump power of $300 \mathrm{~mW}$, the temporal behavior of the pulse train and the single pulse shape are shown in Fig. 6(a) and 6(b), respectively. The duration of pulse is measured to be $17 \mathrm{~ns}$ and the pulse repetition rate is about $0.66 \mathrm{MHz}$.

(a)
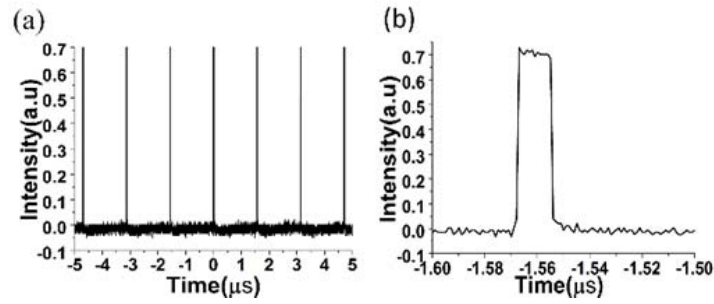

Fig. 6. Mode-locked laser output: (a) Mode-locked pulse sequence; (b) The single pulse shape.

In the mode-locked figure-8 cavity, the MSC functions as a mode converter and splitter, the LP01 mode is converted to LP11 mode when the pulse light passes through the coupling region and outputs the LP11 mode at the TMF output port (output2). Radially polarized and azimuthally polarized beams with a high purity can be obtained at output2 through adjustment of PC2 and PC3. The doughnut-shaped intensity profiles of the radially and azimuthally polarized beams are measured and shown in Figs. 7(a)-7(f). The purity of the radially polarized beam and the azimuthally polarized beam are measured to be $94.2 \%$ and $94.3 \%$, respectively [10]. Figs. 7(b)-7(e) and 7(g)-7(j) show the intensity distributions of the radially polarization beam and azimuthally polarization beam after passing a liner polarizer at different orientations.

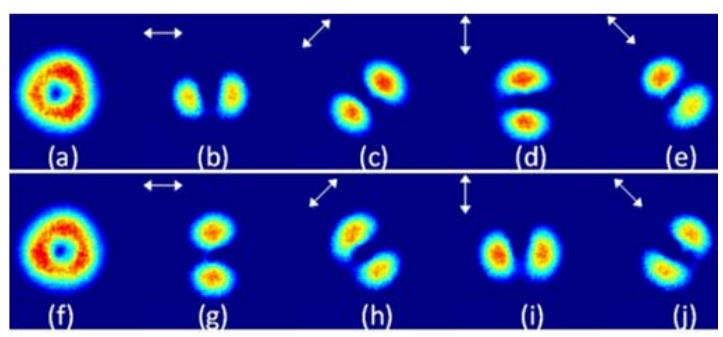

Fig. 7. Intensity distributions of: (a) radially polarization beam and (f) azimuthally polarization beam without a polarizer; (b)-(e) show the intensity distributions of radially polarization beam after passing a liner polarizer; (g)-(j) show the intensity distributions of azimuthally polarization beam after passing a liner polarizer. Arrow indicates the orientation of the linear polarizer.

\section{SUMMARY}

In summary, we present an all-fiber passively mode-locked laser producing CVBs with high efficiency and high mode purity. A MSC made by weakly fused technology is used as the transverse mode converter and mode splitter. The purities of the radially polarized beam and the azimuthally polarized beam are measured to be $94.2 \%$ and $94.3 \%$, respectively. The laser is mode-locked within a spectral bandwidth of 3.2 $\mathrm{nm}$ at the $1556.3 \mathrm{~nm}$ operating wavelength. The repetition rate of the mode-locked laser is $0.66 \mathrm{MHz}$ and the pulse duration is $17 \mathrm{~ns}$.

\section{ACKNOWLEDGMENTS}

This work is partly supported by National Science Foundation of Jiangsu Province (Nos. BK20150858, BK20161521), the Six Talent Peaks Project in Jiangsu Province (No. 2015-XCL-023), the Talents Projects in Nanjing University of Posts and Telecommunications (Nos. NY214002, NY215002).

\section{REFERENCES}

[1] A. Bouhelier, F. Ignatovich, and A. Bruyant, "Surface plasmon interference excited by tightly focused laser beams," Opt. Letter, 32, 17, pp. 2535-2537, Sep.1 2007

[2] R. S. R. Ribeiro, O. Soppera, A. G. Oliva, A. Guerreiro and P. A. Jorge, "New trends on optical fiber tweezers," J. Lightwave Technology, 33, 16, pp. 3394-3405, Aug.15 2015.

[3] D. Lin, K. Xia, and J. Li, "Efficient, high-power, and radially polarized fiber laser,” Opt. Letter, pp. 2290-2292 Jul. 12010

[4] Q. Zhan, "Cylindrical vector beams: from mathematical concepts to applications," Adv. Opt. Photonics, 1, 1, pp. 1-57, Jan. 2009.

[5] H. Wan, H. Li, and C. Wang, "An Injection-Locked Single-Longitudinal-Mode Fiber Ring Laser with Cylindrical Vector Beam Emission," IEEE Photon. Journal, 9, 1, pp. 1-8, Jan.16 2017.

[6] D. Mao, T. Feng, and W. Zhang, "Ultrafast all-fiber based cylindrical-vector beam laser," Appl. Phys. Letter, 110, 2 , pp. 021107, Jan.9 2017.

[7] B. Sun, A. Wang, and C. Gu, "Mode-locked all-fiber laser producing radially polarized rectangular pulses." Opt. Letter, 40, 8, pp. 1691-1694, Apr.15 2015.

[8] K. Yan, J. Lin, and Y. Zhou, "Bi2Te3 based passively Q-switched fiber laser with cylindrical vector beam emission," Appl. Optics, 55, 11, pp. 3026, Apr.10 2016.

[9] R. Ismaeel, T. Lee, and B. Oduro, "All-fiber fused directional coupler for highly efficient spatial mode conversion," Opt. Express, 22, 10, pp. 11610-11619 May. 192014

[10] B. Sun, A. Wang, and L. Xu, "Low-threshold single-wavelength all-fiber laser generating cylindrical vector beams using a few-mode fiber Bragg grating," Opt. Letter, 37, 4, pp, 464-466, Feb.15 2012 